\title{
e-Migrinter
}

$11 \mid 2013$

Et l'immobilité dans la circulation?

\section{À l'origine de ces journées, une série d'interrogations sur les rapports entre mobilité et immobilité}

\section{Françoise Dureau}

\section{CpenEdition}

\section{Journals}

Édition électronique

URL : https://journals.openedition.org/e-migrinter/212

DOI : 10.4000/e-migrinter.212

ISSN : 1961-9685

Éditeur

UMR 7301 - Migrinter

\section{Édition imprimée}

Date de publication : 1 septembre 2013

Pagination : 7-14

ISSN : 1961-9685

\section{Référence électronique}

Françoise Dureau, «À l'origine de ces journées, une série d'interrogations sur les rapports entre mobilité et immobilité », e-Migrinter [En ligne], 11 | 2013, mis en ligne le, consulté le 20 mai 2021. URL: http://journals.openedition.org/e-migrinter/212 ; DOI : https://doi.org/10.4000/e-migrinter.212 
DOSSIER

\section{$1^{\text {ère }}$ partie : exposés introductifs}

\section{À l'origine de ces journées, une série d'interrogations sur les rapports entre mobilité et immobilité}

Françoise Dureau

A

vant d'entrer dans le vif du sujet avec la session introductive où trois des organisateurs (Christophe Imbert, David Lessault, puis Céline Bergeon) vont livrer leurs réflexions à partir de leurs expériences de recherche respectives, quelques mots sur les interrogations qui sont à l'origine de l'atelier (pourquoi ?) et l'organisation des échanges au cours de l'atelier (comment ?).

\section{L'atelier : pourquoi ?}

Cet atelier trouve son origine, entre autres, dans l'identification de biais introduits par le paradigme de la mobilité généralisée (le paradigme «mobilitaire», pour reprendre un vocabulaire à la mode) et la valorisation de la mobilité. Cette situation a pour conséquences : a) de ne cibler que le mouvement, ceux qui bougent, ce qui change; b) d'occulter les permanences (les «stations" selon le vocabulaire de la Time geography (Chardonnel, 2001) et les sédentaires ; c) de dévaloriser les immobiles, a priori considérés comme passifs ${ }^{1}$. On observe pourtant depuis les années 1980, dans les sciences sociales francophones, de nombreuses avancées conceptuelles et méthodologiques. Elles ont permis de mieux décrire les caractéristiques sociales, spatiales et temporelles de la mobilité spatiale et de la circulation des individus ou des groupes sociaux. Elles ont aussi mis en évidence l'importance de l'immobilité et les rapports réciproques entre immobilité et circulation, que ce soit au niveau individuel ou collectif.

La connaissance de la mobilité a en effet bénéficié de trois grands registres

${ }^{1}$ Le modèle de sélectivité de la migration, selon lequel migrer suppose un certain nombre de ressources et de compétences; or, dans certains contextes en changement, c'est rester qui suppose d'avoir plus de moyens et de compétences que partir. 
d'avancées, dans différents champs d'étude, et ce dans différentes régions du globe. Nous en dresserons un rapide panorama, en nous inspirant largement du bilan présenté dans l'ouvrage coordonné par Christophe Imbert (2014). Ce qui permettra de montrer dans quels mouvements scientifiques s'est inscrite cette proposition d'atelier.

D'un point de vue social, un nombre important de recherches ont rattaché la circulation des individus à des logiques collectives, en particulier familiales.

Cette perspective familiale s'exprime dans l'émergence de différentes notions, dans chacun des champs d'étude de la mobilité, en France comme en Afrique de l'Ouest ou en Amérique latine.

Les thèmes de la famille et du logement sont associés depuis longtemps dans la recherche sociologique francophone (Dureau, Imbert, 2014). La notion de «système résidentiel familial» a émergé au début des années 1980 à la fois chez les chercheurs travaillant sur la France (Pinson, 1988) et chez ceux travaillant sur les villes d'Afrique de l'Ouest, où la notion est utilisée pour désigner « un ensemble articulé de lieux de résidence (unités d'habitation) des membres d'une famille étendue ou élargie » (Le Bris et al., 1987 ; Dureau, 2002).

Une notion voisine se retrouve sous la plume de chercheurs latino-américains: le «ménage confédéré » que mobilisent Jorge Balán et Jorge Dandler à propos des Boliviens circulant entre Bolivie et Argentine (Balán, Dandler, 1987). Cette notion s'inscrit dans une approche «classique» en Amérique latine où depuis les années 1960 de nombreuses enquêtes démographiques sur la migration prennent en compte la parentèle non co-résidente. Anthropologues et démographes latino-américains ont montré comment la circulation contribue à assurer la reproduction sociale et économique des familles paysannes.
Dans le champ d'étude de la migration internationale, les travaux ont toujours accordé une place importante à la famille des migrants, en soulignant son rôle dans le choix du lieu de destination et dans l'accueil des migrants, ainsi que l'éclatement géographique des ancrages familiaux; à partir des années 2000, la notion de « famille transnationale », devient très présente dans la production scientifique anglo-saxonne.

Le changement d'unité d'analyse de la mobilité, de l'individu aux unités familiales spatialement éclatées, a largement contribué : - à remettre en cause la vision de la migration comme événement "définitif», substituant un lieu de résidence à un autre ;

- à reconnaitre la diversité des formes de mobilité : il existe une production scientifique importante sur les migrations temporaires et circulaires en Amérique latine depuis un demi-siècle (Reboratti, 1986), et en Afrique de l'Ouest par les travaux de démographes, notamment André Quesnel ;

- à redécouvrir la famille élargie en France : en introduction du numéro spécial de la revue Espaces et sociétés consacré à « $\mathrm{La}$ famille dans tous ses espaces ", Jean-Yves Authier et Catherine Bidou notent ainsi «la persistance de liens forts au sein d'une parenté étendue malgré le processus d'individualisation qui marque la période contemporaine » (Authier, Bidou, 2005). En France comme en Colombie (Dureau, 2002), la famille élargie n'a pas totalement disparu au profit de la famille nucléaire.

D'un point de vue spatial, démographes et géographes ont souligné les limites d'une approche de la mobilité spatiale définie par un lieu de résidence unique.

Dans la plupart des enquêtes démographiques et des recensements, la résidence est définie comme le lieu où l'individu «a coutume d'habiter», le plus souvent considéré comme unique. C'est à travers ce filtre de la résidence unique et permanente que l'analyse de la mobilité 
spatiale a longtemps été limitée aux seules migrations qui correspondent à un transfert de résidence, laissant donc dans l'ombre toutes les autres formes de mobilités temporaires ou circulaires. Les recommandations internationales en matière de statistique démographique continuent d'ailleurs à identifier la multi-résidence comme un problème, jamais comme un objet d'observation et de mesure.

Aux côtés de cette notion de résidence, on observe la présence croissante de notions qui, toutes, reposent sur la reconnaissance du caractère multiple de la localisation des individus et de leur circulation entre les différents lieux constitutifs des espaces de vie. Parmi cellesci, citons :

- l'espace de vie, notion proposée par la géographie sociale dans les années 1970 et reprise dans le champ de la démographie par Daniel Courgeau ;

- l'espace résidentiel ou le système de résidence de l'individu défini par l'ensemble des logements habités par un individu au cours de l'année, l'intensité et le rythme de présence dans chacun d'eux);

- la pluri-résidence, la multi-résidence ou la bi-résidence ;

- plus récemment: la multi-localité, l'habiter polytopique (Stock, 2006) ou l'habiter multilocal, évoqué dans la contribution de Cédric Duchêne-Lacroix.
En Afrique comme en Amérique latine, de nombreux travaux produits dans les trente ou quarante dernières années ont fait progresser, tant sur le plan des concepts que celui des méthodes, l'appréhension des situations résidentielles complexes. C'est tout particulièrement le cas des travaux sur la migration internationale, de ceux qui analysent le marché du travail dans certaines branches d'activités ou de ceux consacrés aux stratégies de survie des familles paysannes. Comme cela a déjà été évoqué, ils ont donné lieu à des observations approfondies des mobilités temporaires et circulaires et à des analyses de la fonction de ces mobilités dans la reproduction sociale et économique des familles.

En France, la bipolarité des pratiques résidentielles et des investissements des migrants internationaux a fait l'objet d'une reconnaissance précoce et partagée, que l'on retrouve par exemple sous l'expression «d'espace de vie transatlantique » employée par Stéphanie Condon (1996) à propos des migrants antillais), ou plus généralement sous le terme «d'espace de vie transnational» des migrants. L'analyse des populations immigrées en France a conduit à remettre en cause sans équivoque la notion de résidence principale, et à analyser au contraire les deux résidences dans leurs complémentarités, leurs relations.

Deux bilans des travaux sur la pluri-résidence peuvent être consultés :

- en 1996, un bilan bibliographique des travaux sur la double résidence a été réalisé par Éliane Nicolino, dans l'ouvrage coordonné par Philippe Bonnin et Roselyne de Villanova, D'une maison l'autre;

- $\quad$ en 2004, deux rencontres ont été organisées par le GIS Ceped, l'Unité de Recherche (UR) 6 de l'Ined et l'UR 13 de l'Ird: dans la première, les participants se sont interrogés sur la manière dont la pluri-résidence est appréhee par les systèmes nationaux de statistiques dans différentes régions du monde. La seconde rencontre a permis de tirer les enseignements de travaux ayant eu pour point commun la reconnaissance de la complexité résidentielle dans différents contextes, en France, en Afrique, en Amérique latine ou en Asie. L'ensemble des contributions aux deux rencontres est disponible dans la collection Les numériques du Ceped (Dureau, Golaz, วกกล) 
Un des résultats des travaux sur la pluri-résidence est que ces états résidentiels complexes peuvent correspondre à des phases de transition (processus de décohabitation; étape préalable à un déménagement, etc.), mais qu'ils peuvent aussi s'inscrire dans la durée. En outre, les transitions familiales et professionnelles tendent à s'étaler dans le temps et à se répéter de plus en plus au long de la vie des individus, plusieurs épisodes de mutation professionnelle ou de vie conjugale pouvant se succéder; d'où la nécessité de capter les durées et les répétitions de ces situations résidentielles complexes.

D'un point de vue temporel, enfin, les approches biographiques de la mobilité ont joué un rôle essentiel dans la mise au jour, pour de nombreux individus, des successions de périodes d'immobilités et de mobilités.

Les premières expériences de recueil de trajectoires migratoires, professionnelles et familiales dans le cadre d'enquêtes démographiques remontent aux années 1960, dans différentes régions du globe: en France, les enquêtes «Peuplement de Paris » et «Mobilité géographique et concentration en France » de l'Ined; en Amérique latine, l'enquête de Monterrey au Mexique; en Afrique de l'Ouest, les enquêtes menées par les démographes de l'Orstom sur le pays Mossi. Après ces recherches pionnières et à la suite de l'enquête «3B-Biographie familiale, professionnelle et migratoire» réalisée par l'Ined en 1981, les collectes biographiques sur la mobilité se multiplient en Europe (selon une méthodologie similaire à celle de l'enquête 3B), puis en Afrique, en Amérique latine et en Asie. Au fil des décennies, le cadre théorique et les méthodes de collecte et d'analyse ont largement progressé.

L'approche transversale - qui considère les événements se produisant à un moment donné - était en vigueur en démographie jusqu'à la fin de la Seconde Guerre mondiale. Dans les années 1950, se mettent en place les principes de l'analyse longitudinale agrégée, par cohortes: l'hypothèse de base réside dans l'indépendance entre les différents phénomènes démographiques, chacun d'eux étant étudié "à l'état pur», dans des populations considérées comme homogènes. Au début des années 1980, une nouvelle approche des comportements individuels fait son apparition en démographie. L'unité d'analyse n'est plus l'événement, mais la biographie individuelle: "Le paradigme, dans ce cas, peut être approché par l'énoncé suivant: un individu parcourt, tout au long de sa vie, une trajectoire complexe, qui dépend, à un instant donné, de sa trajectoire antérieure et des informations qu'il a pu acquérir dans le passé » (Courgeau, 2002, cité par Dureau et Imbert, 2014). Ce changement de paradigme conduit à reconnaître l'interdépendance entre les phénomènes démographiques et à remettre en cause des catégorisations basées sur un événement unique (telles que la catégorisation migrant/non migrant): toute la trajectoire est considérée et l'individu est caractérisé par l'ensemble de son expérience.

En relation avec des changements déjà évoqués, les méthodes de collecte biographique connaissent d'importantes évolutions :

- les comportements individuels de mobilité sont appréhendés dans une approche contextuelle (groupe et autres niveaux de contexte, méso et macro), voire multi-niveaux, qui prend en compte les réseaux de sociabilité des individus ;

- les rapports des individus aux lieux sont considérés en reconnaissant le caractère plurilocal des pratiques spatiales et les relations réciproques existant entre les différentes échelles de mobilité ; 
Deux bilans des expériences de collecte biographique sur la mobilité peuvent être consultés : $-$ 1999: Biographies d'enquêtes, actes d'une rencontre du Groupe de Réflexion sur l'Approche Biographique (GRAB) organisée en 1997, qui a permis de tirer les enseignements de quatorze collectes biographiques réalisées entre 1974 et 1997 dans différentes régions du Monde ;

- $\quad 2013$ : dans le premier chapitre (L’approche biographique des mobilités résidentielles) de l'ouvrage Les circulations métropolitaines (Imbert et alii, 2014), Françoise Dureau et Christophe Imbert ont procédé à une actualisation du bilan dressé dans l'ouvrage du GRAB.

Deux synthèses sur l'approche biographique de la mobilité résidentielle doivent également être mentionnées :

- 2002: État des lieux des recherches sur la mobilité résidentielle en France, réalisé par Catherine Bonvalet et Jacques Brun, dans le premier chapitre de L'accès à la ville;

- $\quad 2010$ : État des connaissances sur les trajectoires résidentielles, dressé sous la direction de Jean-Yves Authier, dans le cadre d'une étude réalisée pour le Plan Urbanisme Construction Architecture (PUCA).

- $\quad$ les trajectoires individuelles, conçues initialement comme une succession d'états binaires définis par des transitions instantanées, sont désormais appréhendées comme des états complexes et comme une combinaison de processus faisant intervenir des temporalités différentes (GRAB, 2006).

Différentes innovations méthodologiques voient le jour, pour collecter une information biographique satisfaisant à ces trois évolutions, notamment pour saisir les situations résidentielles complexes et les circulations entre les pôles des systèmes résidentiels. Ces innovations conduisent à produire une information sur la mobilité à la complexité croissante, que ce soit en termes social, spatial ou temporel: l'enjeu actuel réside de ce fait plus dans le développement des méthodes d'analyse de ce matériau que dans la poursuite de l'amélioration des méthodes de collecte.

L'ensemble des avancées dans l'approche de la mobilité évoquées dans les pages qui précèdent a eu pour effets :

- de mettre en évidence l'importance de l'immobilité : à l'échelle de la vie des individus, les états d'immobilité sont sensiblement plus importants que les moments de déplacement;
- et de montrer que «mobiles» et « immobiles » sont bien loin de constituer des catégories cloisonnées: d'une part, des individus peuvent passer d'un état à l'autre à différentes périodes de leur existence; et, d'autre part, les immobiles prennent autant part à la circulation que les mobiles. La circulation des uns serait alors un moyen d'assurer le maintien des autres en un lieu et, réciproquement, un lieu d'ancrage solide permettrait de pérenniser une pratique de circulation.

Un enjeu actuel important est donc d'essayer de mieux comprendre les rapports entre mobilité et immobilité et les rôles spécifiques des immobiles dans la circulation. D'où l'organisation de ces deux journées d'atelier qui réunissent des chercheurs intéressés par l'exercice qui leur a été proposé: revisiter leurs travaux sous l'angle de l'immobilité et des immobiles.

\section{L'atelier : comment?}

L'organisation de ces journées s'est inscrite dans une double démarche. Il s'agissait tout d'abord de dépasser la segmentation qui marque traditionnellement l'étude de la mobilité (migration internationale, mobilité résidentielle déplacements quotidiens). Mais il s'agissait 
aussi d'aller au-delà de la segmentation existant entre les travaux portant sur la France et ceux sur les pays du Sud, en reconnaissant l'intérêt de l'expérience de la recherche menée dans les pays du Sud à l'égard de certaines pratiques nouvelles ou ignorées par la recherche en France, mais pleinement reconnues ailleurs. L'enjeu était donc de mettre en perspective les avancées produites par différentes disciplines de Sciences sociales, dans différents champs thématiques (migration internationale, mobilité résidentielle et logement, famille) et dans différentes régions du globe (en France, dans d'autres pays européens et dans différents pays du Sud).

Les propositions reçues et sélectionnées ont été regroupées selon quatre thématiques :

1) Circulations, immobilités et cohésion des groupes sociaux ;

2) Circulations et immobilités négociées dans les familles;

3) Circulations et périodes d'immobilité forcée ;

4) Réflexions méthodologiques.

Cette structure thématique traduit la série de questions posée dans l'appel à communications :

- Comment circulation et immobilité se négocient, se partagent et s'organisentelles entre les membres d'un groupe, communautaire ou familial: au cours des différentes sessions de la première journée, différents groupes seront évoqués : la famille nucléaire, la famille élargie, la voisinage, la communauté, le village ;

- Comment circulation et immobilité sont imposées à certains moments, à certains individus: les situations de circulation et d'immobilité forcées seront évoquées pendant la matinée de la deuxième journée ;

- Quelles sont les relations réciproques entre catégorisations et pratiques spatiales, réelles ou perçues, des individus, que ces catégorisations soient le fait du politique, du producteur d'information statistique ou du chercheur : ces questions seront traitées dans la séance méthodologique, au début de la dernière demi-journée de l'atelier.

Les contributions font également apparaître plusieurs séries de questionnements qui se retrouvent dans les différentes sessions de l'atelier. Quatre " fils transversaux » peuvent ainsi être évoqués :

1) le caractère subi et/ou choisi de la mobilité, de la circulation ou de l'immobilité : le degré de contrainte qui pèse sur les pratiques spatiales (mobilité ou immobilité) se retrouve finalement tout au long de l'atelier, et pas seulement dans la session sur l'immobilité forcée ;

2) en partie lié à question précédente, les relations entre mobilité/immobilité et les positions des individus dans les hiérarchies sociales et familiales : quelle que soit l'entrée choisie, on en revient toujours aux positions de l'individu (ou du groupe) dans l'espace et dans le temps, et aux relations entre ces positions (dans le temps et dans l'espace) et les positions dans les hiérarchies sociales et familiales. Les rapports entre genre et mobilité/immobilité sont très présents dans de nombreuses contributions à l'atelier, depuis les familles bourgeoises napolitaines (contribution de Thomas Pfirsch), jusqu'à celles des détenus dans la maison d'arrêt de Nanterre (article de Lucie Bony) ;

3) l'articulation entre les échelles des pratiques spatiales, qu'il s'agisse des échelles spatiales (de l'international à l'intraurbain) ou temporelles (de la vie de l'individu à l'échelle du quotidien): les rapports entre moments de station et de déplacement diffèrent fortement entre les trajectoires résidentielles et les parcours quotidiens (cette différence formelle importante a bien été mise en évidence dans les ateliers du projet «Mobydic » (Mobilités et dynamiques de peuplement : représentations graphiques et production d'indicateurs); plusieurs contributions évoquent les articulations entre mobilités/immobilités résidentielles et quotidiennes au sein de la famille; 
4) en lien avec cette approche multiscalaire des mobilités, la question de la catégorisation des individus et des pratiques spatiales - qui n'est d'ailleurs pas qu'une question de méthode - revient dans la plupart des contributions. En revanche, en dépit de leur importance pour la thématique de l'atelier, deux questions sont peu évoquées en tant que telles: la catégorisation des lieux (un déplacement est un changement de lieu, ce qui pose la question de la limite entre les lieux; on peut également considérer globalement un "type de lieu», qui rassemble des lieux correspondant à des localisations géographiques distinctes mais qui remplissent la même fonction); et la prise en compte de la dynamique des lieux, qui peut conduire les individus à changer de lieu sans effectuer de mobilité spatiale (Dureau et al., 2006).

5) enfin, un danger demeure très présent, celui qui consiste à attribuer aux individus les caractéristiques de leur logement : ce qui conduit à s'interroger plus précisément sur les rapports entre les caractéristiques physiques du logement, son statut légal/illégal et la mobilité/l'immobilité du logement et de ses habitants.
Françoise Dureau

DR IRD honoraire

Migrinter - UMR 7301

f.dureau@gmail.com
Bibliographie

Authier, Jean-Yves (dir.) (2010) Etat des lieux sur les trajectoires résidentielles, Paris, PUCA, $85 \mathrm{p}$.

Authier, Jean-Yves ; Bidou, Catherine (2005) Éditorial : La famille dans tous ses espaces... ou presque!, Espaces et sociétés, n¹20-121, pp. 7-14.

Balán, Jorge ; Dandler Jorge (1987) Marriage process and household formation : migration in the Cochabamba region (Bolivia) and Bolivian migrants in Buenos Aires (Argentina), Colloque L'insertion des migrants dans les villes africaines (10-14 février 1987), Lomé, CRDI - ORSTOM - URD.

Bonnin, Philippe; de Villanova, Roselyne (éd.) (1999) D'une maison l'autre. Parcours et mobilités résidentielles, Paris, Creaphis, 371 p.

Bonvalet, Catherine; Brun, Jacques (2002) État des lieux des recherches sur la mobilité résidentielle en France, in Lévy, J.-P.; Dureau, F. (dir.) L'accès à la ville, les mobil ités spatiales en question, Paris, L'Harmattan, pp. 15-64.

Chardonnel, Sonia (2001) La time-geography : les individus dans le temps et dans l'espace, in Sanders, L. (dir.) Modèles en analyse spatiale, Paris, Editions Hermès, pp. 129-156.

Chombart de Lauwe, Paul-Henry (1960) Famille et habitation, Paris, CNRS éditions, $364 \mathrm{p}$.

Condon, Stéphanie (1996) Les migrants antillais en métropole: un espace de vie transatlantique, Espace, Populations, Sociétés, n²-3, pp. 513-520.

Courgeau, Daniel (2002) Évolution ou révolutions dans la pensée démographique?, Mathématiques et Sciences humaines, 40e année, n¹60, pp. 49-76.

Dureau, Françoise (2002) Les systèmes résidentiels : concepts et applications, in Lévy, J.-P.; Dureau, F. (dir.) L'accès à la ville. Les mobilités spatiales en questions, Paris, L'Harmattan, pp. 355-382. 
Dureau, Françoise; Beauchemin, Cris ; Coubès, Marie-Laure; Delaunay, Daniel (2006) Les mobilités spatiales dans des contextes en évolution: analyse croisée de deux dynamiques, in GRAB, Etats flous et trajectoires complexes. Observation, modélisation, interprétation, Paris, INED, pp. 157-194 (Méthodes et savoirs).

Dureau, Françoise ; Golaz, Valéry (coord.) (2006) Mobilité et résidence, Paris, CEPED [en ligne] URL:

$<\underline{\text { http://www.ceped.org/?Mobilite-et- }}$ residence>

Dureau, Françoise ; Imbert, Christophe (à paraitre 2014) L'approche biographique des mobilités résidentielles, in Imbert, C. ; et alii, D'une métropole à l'autre: pratiques urbaines et circulations dans l'espace européen (perspectives depuis Lisbonne), Paris, Armand Colin (Recherches).

Groupe de réflexion sur l'approche biographique (1999) Biographies d'enquêtes. Bilan de 14 collectes biographiques, Paris, INEDPUF, $340 \mathrm{p}$.

Groupe de réflexion sur l'approche biographique (2006) États flous et trajectoires complexes. Observation, modélisation, interprétation, Paris, INED, $301 \mathrm{p}$.
Imbert, C. ; et alii (à paraître 2014) D'une métropole à l'autre: pratiques urbaines et circulations dans l'espace européen (perspectives depuis Lisbonne), Paris, Armand Colin (Recherches).

Le Bris, Émilie; Marie, Alain; Osmont, Annick; Sinou, Alain (1987) Famille et résidence dans les villes africaines. Dakar, Bamako, Saint-Louis, Lomé, Paris, L'Harmattan, 268 p.

Pinson, Daniel (1988) Du logement pour tous aux maisons en tous genres, Paris, Plan Construction et Architecture, $207 \mathrm{p}$.

Reboratti, Carlos Eduardo (éd.) (1986) Se fue a volver. Seminario sobre las migraciones temporales en América Latina, México, PISPAL/CIUDAD/CENEP, 595 p. 